\title{
Central Limit Theorem for Mixing Quantum Systems and the CCR-Algebra of Fluctuations
}

\author{
D. Goderis ${ }^{\star}$, and P. Vets ${ }^{\star}$ \\ Instituut voor Theoretische Fysica, Universiteit Leuven, B-3030 Leuven, Belgium
}

\begin{abstract}
We analyse macroscopic fluctuations of an infinite quantum system and introduce the CCR-C*-algebra of normal fluctuations. A non-commutative central limit theorem for mixing quantum systems is proved.
\end{abstract}

\section{Introduction}

A macroscopic quantum system is essentially described by two types of observables. The first type being the so-called extensive observables or observables at infinity. From the point of view of probability theory they are obtained as the limit points in the law of large numbers. These limit-observables form a commutative *-algebra. The theory of observables at infinity is by now well understood [1].

The second type of observables are the so-called fluctuation observables or macroscopic fluctuations. Consider the system $(\mathscr{B}, \omega)$, where $\mathscr{B}$ is a quasi-local $C^{*}$-algebra and $\omega$ a state of $\mathscr{B}$. Let $A$ be an observable of the system, then the mathematical problem is to give a rigorous meaning to the limit

$$
\tilde{A}_{\omega}=\lim _{\Lambda \rightarrow \infty} A^{\Lambda}=\lim _{\Lambda \rightarrow \infty} \frac{1}{\Lambda^{1 / 2}} \int_{\Lambda} d x\left(\tau_{x}(A)-\omega\left(\tau_{x}(A)\right)\right),
$$

where $\tau_{x}(A)$ denotes the translation of $A$ by $x \in \mathbb{R}^{v}$.

In $[2,3,4]$ there were attempts to describe these limits. The notion of a system having normal fluctuations was introduced and it was proved that some mean field models satisfy this definition. For these particular models one could compute all higher moments of fluctuations. Recently $[5,6]$ we generalized these results to obtain a model independent theory of fluctuations, i.e. a theory applicable for all mean field models.

In Sect. 3 of this paper we present a theory for more general interacting systems. We redefine the notion of a system $(\mathscr{B}, \omega)$ having normal fluctuations. Instead of requiring the existence of all moments $[2,3,4]$, our definition is based on the notion

\footnotetext{
* Onderzoeker IIKW, Belgium
} 
of characteristic function in probability theory; i.e. the function $\lim _{\Lambda \rightarrow \infty} \omega\left(e^{t t A^{\Lambda}}\right)$ has to be gaussian. For such systems we give a full characterisation of the limit operators $\tilde{A}_{\omega}$. In fact, we prove that the set of all limit operators generates a well defined representation of the CCR-C*-algebra determined by a quasi-free state. The resulting CCR-algebra is called the CCR-algebra of normal fluctuations. The theory contains the earlier results obtained for mean field models.

In Sect. 4 we are looking for a class of systems having normal fluctuations. In probabilistic terms the technical problem we solve is the formulation of a non-commutative central limit theorem (CLT) for mixing systems. There are essentially two methods extending the CLT to the non-commutative case. The first one deals with the momenta $[7,8]$, on which the definition of normal fluctuations given by $[2,3,4]$ is inspired. The second method deals directly with the characteristic function $[5,9]$. The proof of the classical CLT is based on an idea of Bernstein introduced in 1926 [10]. The main condition consists in the $L^{1}$-property of the clusterfunction (or mixing coefficient) $\alpha(t)$, defined on the $\sigma$-algebras $\mathscr{M}_{t_{1}}^{t_{2}}$ induced by the random process $\left(X_{i}\right)_{i=1}^{\infty}$. Finite $(m)$ dependent sequences, i.e. $X_{i}$ and $X_{j}$ are independent for $|i-j|>m$, are examples satisfying this condition. For more details about conditions and results concerning the classical case we refer to [11]. The non-commutative CLT we present in Sect. 4 is also based on the method of Bernstein. However, new techniques are necessary to deal with problems due to the quantum nature of the system $(\mathscr{B}, \omega)$. Remark that for example the set of random variables $\left(\tau_{x}(A)\right)_{x \in \mathbb{R}^{\prime \prime}}$ consists in general of non-commuting observables $\left[\tau_{x}(A), \tau_{y}(A)\right] \neq 0$ for $x \neq y$. The main conditions we impose are clustering conditions on the state $\omega$. We require the $L^{1}$-space clustering of $\omega$ for a norm dense subalgebra $\mathscr{B}_{0}$ of $\mathscr{B}$. This implies the existence of the second moment, i.e. $\lim \omega\left(\left(A^{\Lambda}\right)^{2}\right)<\infty$. We also need a space cluster function $\alpha_{N}^{\omega}(d)$, which measures $\Lambda \rightarrow \infty$

the space-factorization of the state $\omega$. In general this function depends not only on the separation $d$ between two volumes, but also on the size $N$. The condition we impose is a scaling law between $N$ and $d$. If the factorization is supposed to be independent of the size [e.g. 12], then this condition reduces to the classical $L^{1}$-property of the cluster function $\alpha^{\omega}(d)$.

In illustration we give in Sect. 5 a class of states for which the function $\alpha^{\omega}(d)$ has compact support. This example can be seen as a quantum analogue version of the finite dependent sequences in the classical case.

In this paper we restrict ourselves to the case of quantum lattice systems, i.e. the translation group is discrete. The extension to continuous systems should cause no serious difficulties.

\section{Preliminaries about CCR-Algebra and Quasi-Free States}

An essential ingredient in describing the algebra of normal fluctuations of a quantum system $(\mathscr{B}, \omega)$ is the notion of CCR- $C^{*}$-algebra and its quasi-free states. In this section we give the necessary definitions and results. The details about the CCR-algebra can be found in [13], the details about quasi-free states can be found in $[14]$. 
Suppose a real symplectic space $(H, \sigma)$ is given, i.e. $H$ is a real vector space with a (possibly degenerated) symplectic form $\sigma$ defined on it:

$$
\sigma: H \times H \rightarrow \mathbb{R}, \quad(x, y) \rightarrow \sigma(x, y),
$$

such that $\sigma$ is bilinear and anti-symmetric.

Denote by $W(H, \sigma)$ the complex vector space generated by the functions $W(x)$, where $W(x), x \in H$, is defined by

$$
W(x): H \rightarrow \mathbb{C}: y \rightarrow W(x) y=\left\{\begin{array}{lll}
0 & \text { if } & x \neq y \\
1 & \text { if } & x=y
\end{array} .\right.
$$

$W(H, \sigma)$ becomes an algebra with unit $W(0)$ for the product:

$$
W(x) W(y)=W(x+y) e^{-(i / 2) \sigma(x, y)} ; \quad x, y \in H .
$$

$W(H, \sigma)$ is a *algebra for the involution:

$$
W(x) \rightarrow W(x)^{*}=W(-x) .
$$

Finally $W(H, \sigma)$ is a normed *-algebra for the minimal regular norm [15] and we will work with the $C^{*}$-algebra $\overline{W(H, \sigma)}$, which is the completion of $W(H, \sigma)$ for the norm topology. $\overline{W(H, \sigma)}$ is called the CCR-algebra.

A symplectic operator $S$ on the symplectic space $(H, \sigma)$ is a linear operator of $H$ satisfying

$$
\sigma(S x, S y)=\sigma(x, y) \quad \forall x, y \in H .
$$

It is well known [15] that any symplectic operator $S$ defines $a^{*}$-automorphism $\alpha_{s}$ of $\overline{W(H, \sigma)}$ by

$$
\alpha_{S} W(x)=W(S x)
$$

This type of automorphisms are called quasi-free automorphisms.

A state of $\overline{W(H, \sigma)}$ is a positive linear normalized functional of it. The state $\omega$ is called regular if for all $x, y \in H$ the map

$$
\lambda \in \mathbb{R} \rightarrow \omega(W(\lambda x+y))
$$

is continuous. Let $\omega$ be a regular state and let $(\mathscr{H}, \pi, \Omega)$ be the GNS-triplet induced by $\omega$. Then the regularity of $\omega$ implies [15] that there exists a linear map

$$
\begin{aligned}
B: H & \rightarrow \mathscr{L}(\mathscr{H})(\text { linear operators on } \mathscr{H}) \\
x & \rightarrow B(x)
\end{aligned}
$$

such that $\forall x \in H: B(x)=B(x)^{*}$ and $\pi(W(x))=\exp i B(x)$. The map $B$ is called the Bose field, satisfying the Bose field commutation relations:

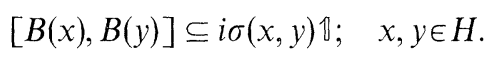

A quasi-free state of $\overline{W(H, \sigma)}$ is a state $\omega_{s}$, with $s$ a real symmetric positive bilinear form of $H$ satisfying

$$
\frac{1}{4}|\sigma(x, y)|^{2} \leqq s(x, x) \cdot s(y, y)
$$


and

$$
\omega_{s}(W(x))=e^{-(1 / 2) s(x, x)} .
$$

Remark that a quasi-free state $\omega_{s}$ is regular.

If $\left(\mathscr{H}_{s}, \pi_{s}, \Omega_{s}\right)$ is the GNS-triplet of $\omega_{s}$ and $B_{s}$ the associated Bose-field, then one easily calculates

$$
\begin{gathered}
\left(\Omega_{s}, B_{s}(x) \Omega_{s}\right)=0, \quad x \in H, \\
\left(\Omega_{s}, B_{s}(x) B_{s}(y) \Omega_{s}\right)=s(x, y)+\frac{i}{2} \sigma(x, y), \quad x, y \in H .
\end{gathered}
$$

\section{The Algebra of Normal Fluctuations}

First we introduce the notion of quasi-local algebras build on a $v$-dimensional lattice $\mathbb{Z}^{v}$. For the general definition of quasi-local algebras, see [16]. Let $\mathscr{D}\left(\mathbb{Z}^{v}\right)$ be the directed set of finite subsets of $\mathbb{Z}^{v}$, where the direction is the inclusion. With each point $x \in \mathbb{Z}^{v}$, we associate the algebra $\mathscr{A}_{x}$, which are all copies of a $C^{*}$-algebra $\mathscr{A}$. For all $\Lambda \in \mathscr{D}\left(\mathbb{Z}^{\nu}\right)$, the tensor product $\bigotimes_{x \in \Lambda} \mathscr{A}_{x}$ defines a $C^{*}$-algebra $\mathscr{A}_{\Lambda}$. If $\mathscr{A}$ is nuclear, then there exists a unique $C^{*}$-norm on $\mathscr{A}_{\Lambda}[17]$, but this restriction is not necessary for our purposes.

The family $\left\{\mathscr{A}_{\Lambda}\right\}_{\Lambda \in \mathscr{P}\left(\mathbb{Z}^{v}\right)}$ has the usual relations of locality and isotony:

$$
\begin{array}{cll}
{\left[\mathscr{A}_{\Lambda_{1}}, \mathscr{A}_{\Lambda_{2}}\right]=0} & \text { if } & \Lambda_{1} \cap \Lambda_{2}=\emptyset, \\
\mathscr{A}_{\Lambda_{1}} \subseteq \mathscr{A}_{\Lambda_{2}} & \text { if } & \Lambda_{1} \subseteq \Lambda_{2} .
\end{array}
$$

Denote by $\mathscr{A}_{L}$ all local observables, i.e.

$$
\mathscr{A}_{L}=\bigcup_{\Lambda \in \mathscr{D}\left(\mathbb{Z}^{v}\right)} \mathscr{A}_{\Lambda} .
$$

Finally the norm closure of $\mathscr{A}_{\Lambda}$ is a quasi-local $C^{*}$-algebra, denoted by

$$
\mathscr{B}=\overline{\cup_{\Lambda} \mathscr{A}_{\Lambda}} \text {. }
$$

Typical examples of such systems are spin-systems, i.e. where $\mathscr{A}$ is a matrix-algebra.

The group $\mathbb{Z}^{v}$ of space translations is a subgroup of the automorphism group of $\mathscr{B}$, whose action we denote by

$$
A \in \mathscr{A}_{\Lambda} \rightarrow \tau_{x}(A) \in \mathscr{A}_{\Lambda+x} ; \quad x \in \mathbb{Z}^{v} .
$$

In the following we consider the system $(\mathscr{B}, \omega)$, with $\omega$ a translation invariant state on $\mathscr{B}$. It is well known [16] that this system satisfies the cluster properties:

(i) asymptotic abelian with respect to space translations: i.e.

$$
\lim _{|x| \rightarrow \infty}\left\|\left[A, \tau_{x} B\right]\right\|=0 \text { for } A, B \in \mathscr{B},
$$

(ii) if $\omega$ is a factor state then

$$
\lim _{|x| \rightarrow \infty}\left|\omega\left(A \tau_{x} B\right)-\omega(A) \omega(B)\right|=0 \quad \text { for } \quad A, B \in \mathscr{B},
$$


(iii) if $\omega$ is a locally normal factor state on $\mathscr{B}$, then for all $\varepsilon>0$, for all $\Lambda \in \mathscr{D}\left(\mathbb{Z}^{v}\right)$, there exists a $\Lambda^{\prime} \in \mathscr{D}\left(\mathbb{Z}^{v}\right)$ such that:

$$
\sup _{\substack{A \in \mathscr{S}^{\prime}, A\left|=1 \\ B \in \mathcal{A}^{\prime},\right| B \mid=1}}|\omega(A B)-\omega(A) \omega(B)| \leqq \varepsilon
$$

for all $\tilde{\Lambda} \in \mathscr{D}\left(\mathbb{Z}^{v}\right)$ with $\tilde{\Lambda} \cap \Lambda^{\prime}=\varnothing$,

(iv) $\mathscr{B}$ is $L^{1}\left(\mathscr{A}_{L}\right)$ asymptotic abelian for space-translations:

$$
\sum_{x \in \mathbb{Z}^{v}}\left\|\left[A, \tau_{x} B\right]\right\|<\infty \text { for } A, B \in \mathscr{A}_{L} .
$$

At this point we are able to introduce fluctuations of the system $(\mathscr{B}, \omega)$. Denote by $\Lambda_{n}$ the cube centered around the origin with edges of length $2 n+1$. Define the partial sums

$$
A^{n}=\frac{1}{\left|\Lambda_{n}\right|^{1 / 2}} \sum_{x \in \Lambda_{n}} \tau_{x}(A-\omega(A)) ; \quad A \in \mathscr{B}
$$

The whole problem is to give a rigorous meaning to the limits $\lim A^{n}=\tilde{A}_{\omega}$. These limits are called macroscopic fluctuations of the system $(\mathscr{B}, \omega)$. In $[2,3]$ Hepp and Lieb suggested that the fluctuations of one point observables $\left(A \in \mathscr{A}_{0}\right)$ form some abstract Lie-algebra such that their commutator is a $c$-number:

$$
\lim _{n \rightarrow \infty}\left[A^{n}, B^{n}\right]=\left[\tilde{A}_{\omega}, \tilde{B}_{\omega}\right]=c_{\omega},
$$

where the limit has to be specified. In the next proposition we make this statement mathematically clear. Denote by $\left(\mathscr{H}_{\omega}, \pi_{\omega}, \Omega_{\omega}\right)$ the GNS-triplet of $(\mathscr{B}, \omega)$. Let $Z_{\omega}$ denote the center of the von Neumann algebra $\pi_{\omega}(\mathscr{B})^{\prime \prime}$. Then we have:

Proposition 3.1. Let $\omega$ be a translation invariant state on $\mathscr{B}$. Consider a norm dense subalgebra $\mathscr{B}_{0}$ of $\mathscr{B}$ such that $\mathscr{B}$ is $L^{1}\left(\mathscr{B}_{0}\right)$ asymptotic abelian for space translations. Then

$$
w(\text { eak })-\lim _{n \rightarrow \infty}\left[A^{n}, B^{n}\right] \in Z_{\omega} \text { for all } A, B \in \mathscr{B}_{0} .
$$

Hence if $\omega$ is a factor state,

$$
w-\lim _{n \rightarrow \infty}\left[A^{n}, B^{n}\right]=c_{\omega}(A, \mathrm{~B}) \mathbb{1}_{\omega}
$$

with

$$
c_{\omega}(A, B)=\lim _{n \rightarrow \infty} \omega\left(\left[A^{n}, B^{n}\right]\right) .
$$

Proof. As $\mathscr{B}$ is $L^{1}\left(\mathscr{B}_{0}\right)$ asymptotic abelian, it follows that

$$
\sum_{y \in Z^{v}}\left[A, \tau_{y} B\right] \in \mathscr{B}, \text { for all } A, B \in \mathscr{B}_{0} .
$$

Hence

$$
w-\lim _{n \rightarrow \infty} \frac{1}{\left|\Lambda_{n}\right|} \sum_{x \in \Lambda_{n}} \tau_{x}\left(\sum_{y \in Z^{v}}\left[A, \tau_{y} B\right]\right) \in Z_{\omega} .
$$


An easy computation yields:

$$
\begin{aligned}
\omega-\lim _{n \rightarrow \infty}\left[A^{n}, B^{n}\right] & =\omega-\lim _{n \rightarrow \infty} \frac{1}{\left|\Lambda_{n}\right|} \sum_{x, y \in \Lambda_{n}}\left[\tau_{x}(A), \tau_{y}(B)\right] \\
& =\omega-\lim _{n \rightarrow \infty} \frac{1}{\left|\Lambda_{n}\right|} \sum_{x \in \Lambda_{n}} \tau_{x}\left(\sum_{y \in \Lambda_{n}}\left[A, \tau_{y-x}(B)\right]\right) \\
& =\omega-\lim _{n \rightarrow \infty} \frac{1}{\left|\Lambda_{n}\right|} \sum_{x \in \Lambda_{n}} \tau_{x}\left(\sum_{y \in Z^{v}}\left[A, \tau_{y} B\right]\right),
\end{aligned}
$$

where in the last step we used again the asymptotic abelian condition. This proves the proposition.

Proposition 3.1 states that the commutator of two fluctuations behave like bosons. This phenomenon has been remarked several times $[2,3,7]$. However, Proposition 3.1 does not give a rigorous meaning to the limits $\lim A^{n}=\tilde{A}_{\omega}$ themselves. Recently we solved the problem for mean-field systems, i.e. $\omega$ is a product state $[5,6]$. We showed that the set of all limit operators $\tilde{A}_{\omega}$ with $A$ a self-adjoint one point observable, generates a well defined representation of the CCR-C* algebra, determined by a quasi-free state. A generalization of these methods suggests the following definition.

Definition 3.2. Define $s_{n}(A, B)=\operatorname{Re} \omega\left(A^{n} B^{n}\right)$ for all self adjoint elements $A, B \in \mathscr{B}$. Let $\mathscr{B}_{0}$ be a ${ }^{*}$-subalgebra of $\mathscr{B}$. The system $\left(\mathscr{B}, \mathscr{B}_{0}, \omega\right)$ has normal fluctuations if

$$
\begin{aligned}
& \text { 1. } \forall A, B \in \mathscr{B}_{0}: \sum_{x \in Z^{\nu}}\left|\omega\left(A \tau_{x} B\right)-\omega(A) \omega(B)\right|<\infty . \\
& \text { 2. } \forall A=A^{*} \in \mathscr{B}_{0}: \lim _{n \rightarrow \infty} \omega\left(e^{\imath t A^{n}}\right)=e^{\left.-(1 / 2) t^{2}{ }^{2}(A) A\right)}
\end{aligned}
$$

with

$$
s_{\omega}(A, B)=\lim _{n \rightarrow \infty} s_{n}(A, B) ; \quad A, B \text { s.a. elements of } \mathscr{B}_{0} .
$$

Remark that $\lim _{n \rightarrow \infty} s_{n}(A, B)$ exists by condition 1 . Now we can define the algebra of normal fluctuations of a system $\left(\mathscr{B}, \mathscr{B}_{0}, \omega\right)$ satisfying Definition 3.2. For each such system we construct in a canonical way a CCR- $C^{*}$-algebra $W(H, \sigma)$. Therefore we have to fix the symplectic space $(H, \sigma)$.

Denote by $\mathscr{B}_{0, \text { sa }}$ the real vectorspace of self-adjoint elements of $\mathscr{B}_{0}$. Define for all $A, B \in \mathscr{B}_{0 \text {,sa }}$

$$
\sigma_{n}(A, B)=-i \omega\left(\left[A^{n}, B^{n}\right]\right) .
$$

Take now

$$
\begin{aligned}
& H=\mathscr{B}_{0 . \mathrm{sa}}, \\
& \sigma_{\omega}(A, B): H \times H \rightarrow \mathbb{R} \\
&:(A, B) \rightarrow \sigma_{\omega}(A, B)=\lim _{n \rightarrow \infty} \sigma_{n}(A, B) .
\end{aligned}
$$


Again $\lim \sigma_{n}(A, B)$ exists by condition 1 of Definition 3.2. and clearly $\sigma_{\omega}(.,$.$) defines$ a symplectic form on $\mathscr{B}_{0, \mathrm{sa}}$. The CCR-C $C^{*}$-algebra $\overline{W\left(\mathscr{B}_{0, \mathrm{sa}}, \sigma_{\omega}\right)}$ is called the algebra of normal fluctuations of the system $\left(\mathscr{B}, \mathscr{B}_{0}, \omega\right)$.

Theorem 3.3. If $\left(\mathscr{B}, \mathscr{B}_{0}, \omega\right)$ has normal fluctuations then the limits $\lim _{n \rightarrow \infty} \omega\left(e^{i A^{n}}\right)=$ $e^{-(1 / 2) s_{\omega}(A, A)}, A \in \mathscr{B}_{0, \text { sa }}$ define a quasi-free state $\omega_{s}$ of the CCR-C*-algebra of normal fluctuations $\overline{W\left(\mathscr{B}_{0, \mathrm{sa}}, \sigma_{\omega}\right)}$ by

$$
\omega_{s}(W(A))=e^{-(1 / 2) s_{\omega}(A, A)} .
$$

Moreover if $\alpha$ is $a^{*}$-automorphism of $\mathscr{B}$ such that $\mathscr{B}_{0}$ is $\alpha$-invariant, $\omega(\alpha(A))=\omega(A)$ for all $A \in \mathscr{B}$ and $\left[\alpha, \tau_{x}\right]=0$ for all $x \in Z^{v}$ then $\tilde{\alpha}(W(A))=W(\alpha(A))$ defines a quasi-free *-automorphism of $\overline{W\left(\mathscr{B}_{0, \mathrm{sa}}, \sigma_{\omega}\right)}$

Proof. As $s_{\omega}(A, B)=\lim _{n \rightarrow \infty} \operatorname{Re} \omega\left(A^{n}, B^{n}\right)$ it is obvious that $s_{\omega}$ defines a real, symmetric positive bilinear form on $\overline{W\left(\mathscr{B}_{0, \mathrm{sa}}, \sigma_{\omega}\right)}$. Thus (3.14) defines a quasi-free state if the relation (2.5), i.e. $\frac{1}{4}\left|\sigma_{\omega}(A, B)\right|^{2} \leqq s_{\omega}(A, A) s_{\omega}(B, B)$, holds. For each $n \in \mathbb{N}_{0}$ it follows from Schwartz-inequality:

$$
\begin{aligned}
\frac{1}{4}\left|\sigma_{n}(A, B)\right|^{2} & =\frac{1}{4}\left|\omega\left(\left[A^{n}, B^{n}\right]\right)\right|^{2}=\left|\operatorname{Im} \omega\left(A^{n} B^{n}\right)\right|^{2} \\
& \leqq \omega\left(\left(A^{n}\right)^{2}\right) \omega\left(\left(B^{n}\right)^{2}\right)=s_{n}(A, A) s_{n}(B, B),
\end{aligned}
$$

and (2.5) follows by taking the limit. This proves the first statement. To prove the second statement we have to show that $\alpha$ defines a symplectic operator (2.2) on $\left(\mathscr{B}_{0, \mathrm{sa}}, \sigma_{\omega}\right)$. But for all $n \in \mathbb{N}_{0}$ :

$$
\begin{aligned}
(\alpha A)^{n} & =\frac{1}{\left|\Lambda_{n}\right|^{1 / 2}} \sum_{x \in \Lambda_{n}} \tau_{x}(\alpha A-\omega(\alpha A)) \\
& =\frac{1}{\left|\Lambda_{n}\right|^{1 / 2}} \sum_{x \in \Lambda_{n}} \alpha\left(\tau_{x}(A)-\omega(A)\right)=\alpha\left(A^{n}\right) .
\end{aligned}
$$

Hence

$$
\begin{aligned}
\sigma_{n}(\alpha A, \alpha B) & =-i \omega\left(\left[(\alpha A)^{n},(\alpha B)^{n}\right]\right) \\
& =-i \omega\left(\alpha\left[A^{n}, B^{n}\right]\right)=\sigma_{n}(A, B) .
\end{aligned}
$$

The theorem follows by taking the limit.

Remarks.

1. In $[5,6]$ we considered mean-field models. As the equilibrium states of such models are product states [18], it is sufficient to consider one point fluctuations, i.e. $\mathscr{B}_{0}=\mathscr{A}_{0}$. For such systems we showed that Definition (3.2) is satisfied $[5,6]$.

2. In $[2,3,4]$ the authors give another definition of a system $(\mathscr{B}, \omega)$ having normal fluctuations. The condition on the characteristic functions (3.11) is replaced by the existence of all higher moments: $\lim _{n \rightarrow \infty} \omega\left(\left(A^{n}\right)^{m}\right)<\infty$ for all $m \in \mathbb{N}$. It can be shown that if all moments exist and have a gaussian (or 
normal) behaviour, then condition (3.11) is satisfied. Furthermore as they are dealing with mean field models, they only consider one point fluctuations. However, for mixing quantum systems $\mathscr{B}_{0}$ should contain the norm dense subalgebra $\mathscr{A}_{L}$.

3. Consider the GNS-triplet $\left(\mathscr{H}_{s}, \pi_{s}, \Omega_{s}\right)$ of $\overline{W\left(\mathscr{B}_{0, \mathrm{sa}}, \sigma_{\omega}\right)}$ induced by the quasi-free state $\omega_{s}$ defined in Theorem 3.3. By the regularity of $\omega_{s}$ one can define the Bose field $B_{s}$. Theorem 3.3 states that the macroscopic fluctuations $\tilde{A}_{\omega}$ can indeed by regarded as boson fields, satisfying the CCR-relations

$$
\left[\tilde{A}_{\omega}, \widetilde{B}_{\omega}\right]=\left[B_{s}(A), B_{s}(B)\right] \subseteq i \sigma_{\omega}(A, B) \mathbb{1}_{s} .
$$

This is in agreement with Proposition 3.1.

4. The last part of Theorem 3.3 gives an interesting method for studying the original system $(\mathscr{B}, \omega)$ by means of the properties of the fluctuation algebra. For example if one has a time evolution $\left(\alpha_{t}\right)_{t \in \mathbb{R}}$ generated by a translation invariant hamiltonian, if the state $\omega$ is a $\alpha_{t}$-invariant state and $\mathscr{B}_{0}$ is large enough, i.e. $\alpha_{t} \mathscr{B}_{0} \subseteq \mathscr{B}_{0}$, then $\left(\alpha_{t}\right)_{t \in \mathbb{B}}$ satisfies the conditions of Theorem 3.3. Hence, $\alpha_{t}$ induces a quasi-free time evolution $\tilde{\alpha}_{t}$ on $\bar{W}_{\left(\mathscr{B}_{0, \mathrm{sa}}, \sigma_{\omega}\right)}$, which describes the evolution of the fluctuations by $\tilde{\alpha}_{t}\left(\tilde{A}_{\omega}\right)=\left(\tilde{\alpha}_{t} A\right)_{\omega}$. The quasi-free evolution is already studied for mean-field models $[5,6]$. At another occasion we return to the physical applications in other models.

\section{Central Limit Theorem for Mixing Quantum Systems}

In the foregoing section we introduced the system $\left(\mathscr{B}, \mathscr{B}_{0}, \omega\right)$ and defined under which conditions this system has normal fluctuations. These conditions (3.11) state that the characteristic function $\omega\left(e^{i t A^{n}}\right)$ has to converge pointwise to a gaussian functional on $\mathscr{B}_{0, \mathrm{sa}}$. In probability theory this means that the central limit theorem holds for all random variables $A \in \mathscr{B}_{0, \mathrm{sa}}$.

The aim of this section is to construct reasonable cluster conditions such that (3.11) is satisfied. As we adapt the method of Bernstein used in the classical central limit theorem, our conditions are partially inspired by the classical case $[10,11,19]$. On the other hand conditions with a typical quantum nature arise, such as the $L^{1}$-asymptotic abelianness (CL 1) and the condition (CL 5) on the cluster functions $\alpha_{N}^{\omega}($.$) , which we will introduce now.$

Let $(\mathscr{B}, \omega)$ be the system introduced in Sect. 3. Property (3.6) of quasi-local algebra states that

$$
\sup _{\substack{A \in \mathscr{A}_{A}, A=1 \\ B \in \mathcal{A}_{\tilde{A}}, B:=1}}|\omega(A B)-\omega(A) \omega(B)|
$$

tends to zero as the distance $d(\Lambda, \tilde{\Lambda})$ between $\Lambda$ and $\tilde{\Lambda}$ tends to infinity. In general this quantity is not only dependent on the separation between $\Lambda$ and $\tilde{\Lambda}$, but also on their shape. Therefore, we introduce, for all $N \in \mathbb{N}_{0}, d \in \mathbb{R}^{+}$the cluster function.

$$
\alpha_{N}^{\omega}(d)=\sup _{\Lambda, \tilde{\Lambda}}\left\{\left(\sup _{\substack{A \in \mathscr{A}_{A}, A \\ B \in \mathscr{S}^{\prime}, B^{\prime}=1}}|\omega(A B)-\omega(A) \omega(B)|\right): \Lambda, \tilde{\Lambda} \in \mathscr{D}\left(\mathbb{Z}^{v}\right)\right.
$$




$$
\text { such that } d(\Lambda, \tilde{\Lambda}) \geqq d \text { and } \max (|\Lambda|,|\tilde{\Lambda}|) \leqq N\} \text {. }
$$

From the definition it is obvious that

$$
\alpha_{N}^{\omega}(d) \leqq \alpha_{N}^{\omega}\left(d^{\prime}\right) \text { for } \quad N \in \mathbb{N}_{0} \quad \text { and } \quad d \geqq d^{\prime}
$$

and

$$
\alpha_{N}^{\omega}(d) \leqq \alpha_{N^{\prime}}^{\omega}(d) \text { for } \quad d \in \mathbb{R}_{0} \text { and } \quad N \leqq N^{\prime} .
$$

Moreover property (3.6) implies also

$$
\lim _{d \rightarrow \infty} \alpha_{N}^{\omega}(d)=0 \text { for all } N \in \mathbb{N}_{0} .
$$

Now let $\left(\mathscr{B}, \mathscr{B}_{0}, \omega\right)$ be a system as defined in Sect. 3. The cluster conditions can be formulated as follows:

CL 1: $\mathscr{B}$ is $L^{1}\left(\mathscr{B}_{0}\right)$ asymptotic abelian,

CL 2: for all $A \in \mathscr{B}_{0}$, there exists an $\varepsilon_{1}>0$ and a sequence $\left(\Gamma_{N}, A_{N}\right)_{N \in \mathbb{N}_{0}}$ such that

$$
\lim _{N \rightarrow \infty}\left|\Gamma_{N}\right|^{1+\varepsilon_{1}}\left\|A-A_{N}\right\|<\infty,
$$

where $A_{N} \in \mathscr{A}_{\Gamma_{N}}$ and $\Gamma_{N}$ is a cube with edges of length $N$,

CL 3: $\left(\mathscr{B}, \mathscr{B}_{0}, \omega\right)$ satisfies condition (3.10), i.e. $\omega$ is a $L^{1}$-clustering state, of $\mathscr{B}_{0}$

CL 4: for all $A \in \mathscr{B}_{0, \mathrm{sa}}$ there exists an $\varepsilon_{2}>0$ such that

$$
\lim _{n \rightarrow \infty} \frac{1}{\left|\Lambda_{n}\right|^{1-\varepsilon_{2}}} \omega\left(\left(A^{n}\right)^{4}\right)<\infty,
$$

CL 5: there exists a $\delta>0$ such that:

$$
\lim _{N \rightarrow \infty} N^{1 / 2} \alpha_{N}^{\omega}\left(N^{(1 / 2 v)-\delta}\right)=0 .
$$

Theorem 4.1. If the system $\left(\mathscr{B}, \mathscr{B}_{0}, \omega\right)$ satisfies the cluster conditions $(C L 1 \rightarrow 5)$, then it has normal fluctuations, i.e.

$$
\lim _{n \rightarrow \infty} \omega\left(e^{i t A^{n}}\right)=e^{-(1 / 2) t^{2} s_{\omega}(A . A)} ; \quad A \in \mathscr{B}_{0, \mathrm{sa}},
$$

where $A^{n}$ and $s_{\omega}(.,$.$) are respectively defined in (3.8) and (3.12).$

Before proving this theorem, let us first make some comments on the cluster conditions.

Clearly $(C L 1,2)$ are conditions on the subalgebra $\mathscr{B}_{0}$, expressing that the elements of $\mathscr{B}_{0}$ have to be "local enough." For example if $\mathscr{B}_{0}=\mathscr{A}_{L}$, then $(\mathrm{CL} 1,2)$ are trivially fulfilled.

Condition (CL 3) is essential because it guarantees the existence of the second moment, i.e. $\lim _{N \rightarrow \infty} \omega\left(A^{n}\right)^{2}<\infty$ for all $A \in \mathscr{B}_{0}$. This condition occurs also in the classical central limit theorem for mixing random variables.

(CL4) indicates that the existence of the fourth moment $\left(\varepsilon_{2}=1\right)$ is not necessary to prove the theorem. In fact (CL 4) is a very weak condition, because by (CL 3) 


$$
\begin{aligned}
\lim _{n \rightarrow \infty} \frac{\omega\left(\left(A^{n}\right)^{4}\right)}{\left|\Lambda_{n}\right|} & \leqq \lim _{n \rightarrow \infty} \frac{\left(\left\|A^{n}\right\|^{2}\right)}{\left|\Lambda_{n}\right|} \omega\left(\left(A^{n}\right)^{2}\right) \\
& \leqq \lim _{n \rightarrow \infty} \omega\left(\left(A^{n}\right)^{2}\right) \cdot\|A\|<\infty \text { for all } A=A^{*} \in \mathscr{B}_{0} .
\end{aligned}
$$

It is useful to compare (CL 5) with the classical case. In classical probability theory, given a sequence of random variables $\left(x_{i}\right)_{i \in \mathbb{Z}}(v=1)$, one has the disposal of a uniform clusterfunction. In our notations this should mean that $\alpha_{N}^{\omega}($.$) is independent of N$, or the clustering depends only on the separation between the volumes. This assumption was also made in [12], where $\alpha^{\omega}($.$) is called the modulus of decoupling.$ In this situation (CL 5) is equivalent to

$$
\alpha^{\omega}(d)=0\left(\frac{1}{d^{v+\delta}}\right), \quad \delta>0,
$$

implying that $\alpha^{\omega}(d) \in L^{1}\left(\mathbb{R}^{+}, d x\right) ; v=1,2,3, \ldots$.

This corresponds to the uniform mixing condition in the classical central limit theorem [11, Theorem 18.5.4]. In general however one cannot expect uniform clustering. Therefore condition (4.5) is replaced by a scaling law.

Proof of Theorem 4.1. For notational convenience we write out the proof in full detail in one dimension, i.e. $v=1$. Afterwards we discuss the general case, i.e. $v \in \mathbb{N}_{0}$. Also without loss of generality we suppose $A \in \mathscr{B}_{0}$ such that $\omega(A)=0$. Define for all $n \in \mathbb{N}_{0}$,

$$
\begin{aligned}
& p(n)=\left[(2 n+1)^{1 / 2} \log (2 n+1)\right], \\
& q(n)=\left[\frac{2 n+1}{p(n)}\right], \\
& k(n)=\left[\frac{2 n+1}{p(n)+q(n)}\right],
\end{aligned}
$$

where $[x]$ denotes the integer part of $x \in \mathbb{R}^{+}$. We consider the following quantities:

$$
\begin{aligned}
& \xi_{0}^{n}(A)=\frac{1}{(2 n+1)^{1 / 2}} \sum_{x=-n}^{-n+p-1} \tau_{x}(A), \\
& \eta_{0}^{n}(A)=\frac{1}{(2 n+1)^{1 / 2}} \sum_{x=-n+p}^{-n+(p+q)-1} \tau_{x}(A), \\
& \xi_{i}^{n}(A)=\tau_{i(p+q)} \xi_{0}^{n}(A) \quad 0 \leqq i \leqq k-1, \\
& \eta_{i}^{n}(A)=\tau_{i(p+q)} \eta_{0}^{n}(A) \quad 0 \leqq i \leqq k-1, \\
& \eta_{k}^{n}(A)=\frac{1}{(2 n+1)^{1 / 2}} \quad \sum_{x=-n+k(p+q)} \tau_{x}(A), \\
& S_{n}^{\prime}(A)=\sum_{i=0}^{k-1} \xi_{i}^{n}(A), \\
& S_{n}^{\prime \prime}(A)=\sum_{i=0}^{k} \eta_{i}^{n}(A) .
\end{aligned}
$$


Remark that $A^{n}=S_{n}^{\prime}(A)+S_{n}^{\prime \prime}(A)$. We usually denote $\xi_{0}^{n}, \ldots$ instead of $\xi_{0}^{n}(A), \ldots$. The theorem is proved by the following sequence of observations. Observation 1:

$$
\lim _{n \rightarrow \infty}\left|\omega\left(e^{i t A^{n}}\right)-\omega\left(e^{i t S_{n}^{\prime}(A)}\right)\right|=0 .
$$

Using a well known integral formula:

$$
e^{i t A^{n}}=e^{i t\left(S_{n}^{\prime}+S_{n}^{\prime \prime}\right)}=e^{i t S_{n}^{\prime}}+\int_{0}^{t} d s e^{i s S_{n}^{\prime}} S_{n}^{\prime \prime} e^{i(t-s)\left(S_{n}^{\prime}+S_{n}^{\prime \prime}\right)}
$$

Hence

$$
\begin{aligned}
\mid \omega\left(e^{i t A^{n}}\right)-\omega\left(e^{\left.i t S_{n}^{\prime}\right)} \mid\right. & \leqq \int_{0}^{t} d s\left|\omega\left(e^{i s S_{n}^{\prime}} S_{n}^{\prime \prime} e^{i(t-s)\left(S_{n}^{\prime}+S_{n}^{\prime \prime}\right)}\right)\right| \\
& \leqq \int_{0}^{t} d s\left|\omega\left(\left[e^{i s S_{n}^{\prime}}, S_{n}^{\prime \prime}\right] e^{i(t-s)\left(S_{n}^{\prime}+S_{n}^{\prime \prime}\right)}\right)\right| \\
& +\int_{0}^{t} d s\left|\omega\left(S_{n}^{\prime \prime} e^{i s S_{n}^{\prime}} e^{i(t-s)\left(S_{n}^{\prime}+S_{n}^{\prime \prime}\right)}\right)\right| .
\end{aligned}
$$

As $A=A^{*}$ we obtain by Schwarz inequality

$$
\begin{aligned}
\left|\omega\left(e^{i t A^{n}}\right)-\omega\left(e^{i t S_{n}^{\prime}}\right)\right| & \leqq \int_{0}^{t}\left\|\left[e^{i s S_{n}^{\prime}}, S_{n}^{\prime \prime}\right]\right\| d s+\int_{0}^{t} d s\left|\omega\left(\left(S_{n}^{\prime \prime}\right)^{2}\right)\right|^{1 / 2} \\
& \leqq \int_{0}^{t} d s s\left\|\left[S_{n}^{\prime}, S_{n}^{\prime \prime}\right]\right\|+t\left(\omega\left(S_{n}^{\prime \prime}\right)\right)^{1 / 2} \\
& \leqq \frac{t^{2}}{2}\left\|\left[S_{n}^{\prime}, S_{n}^{\prime \prime}\right]\right\|+t\left(\omega\left(S_{n}^{\prime \prime 2}\right)\right)^{1 / 2}
\end{aligned}
$$

where we used the bound for $A=A^{*}:\left\|\left[e^{i A}, B\right]\right\| \leqq\|[A, B]\|(*)$. The proof of this bound is postponed until the end.

$$
\text { By }(\mathbf{C L} \mathbf{1}) \text { we have } \lim _{n \rightarrow \infty}\left\|\left[S_{n}^{\prime}, S_{n}^{\prime \prime}\right]\right\|=0 \text { : }
$$

Indeed

$$
\begin{aligned}
& \left\|\left[S_{n}^{\prime}, S_{n}^{\prime \prime}\right]\right\| \leqq \sum_{i=0}^{k-1} \sum_{j=0}^{k}\left\|\left[\xi_{i}^{n}, \eta_{j}^{n}\right]\right\| \\
& \leqq \sum_{i=0}^{k-1} \sum_{j=0}^{k-1}\left\|\left[\xi_{0}^{n}, \tau_{(j-i)(p+q)} \eta_{0}^{n}\right]\right\|+\sum_{i=0}^{k-1}\left\|\left[\xi_{i}^{n}, \eta_{k}^{n}\right]\right\| \\
& \leqq k \sum_{i=-k+1}^{k-1}\left\|\left[\xi_{0}^{n}, \tau_{i(p+q)} \eta_{0}^{n}\right]\right\|+\sum_{i=0}^{k-1}\left\|\left[\xi_{i}^{n}, \eta_{k}^{n}\right]\right\| \\
& \left\|\left[S_{n}^{\prime}, S_{n}^{\prime \prime}\right]\right\| \leqq \frac{k}{(2 n+1)} \sum_{i=-k+1}^{k-1} \sum_{x=-n}^{-n+p-1} \sum_{y=-n+p}^{-n+(p+q)-1}\left\|\left[A, \tau_{i(p+q)+y-x} A\right]\right\| \\
& +\frac{1}{(2 n+1)} \sum_{l=0}^{k-1} \sum_{x=-n}^{-n+p-1} \sum_{y=-n+k(p+q)}^{n}\left\|\left[A, \tau_{i(p+q)+v-x} A\right]\right\|
\end{aligned}
$$


as

$$
\leqq \sum_{x=-\infty}^{+\infty}\left\|\left[A, \tau_{x} A\right]\right\|\left(0\left(\frac{k q}{2 n+1}\right)+0\left(\frac{p}{2 n+1}\right)\right)
$$

and

$$
\sum_{x=-\infty}^{\infty}\left\|\left[A, \tau_{x} A\right]\right\|<\infty, 0\left(\frac{p}{2 n+1}\right)=0\left(\frac{\log 2 n+1}{(2 n+1)^{1 / 2}}\right) \rightarrow 0
$$

$$
0\left(\frac{k q}{2 n+1}\right)=0\left(\frac{q}{p+q}\right)=0\left(\frac{q}{p}\right)=0\left(\frac{1}{(\log 2 n+1)^{2}}\right) \rightarrow 0 .
$$

Equation (4.7) follows. Analogously (CL 3) implies

$$
\begin{gathered}
\lim _{n \rightarrow \infty} \omega\left(S_{n}^{\prime \prime 2}\right)=0 \\
\omega\left(S_{n}^{\prime \prime 2}\right)=\sum_{i \neq j=0}^{k-1} \omega\left(\eta_{i}^{n} \eta_{j}^{n}\right)+\sum_{i=0}^{k-1} \omega\left(\left(\eta_{i}^{n}\right)^{2}\right)+\sum_{i=0}^{k-1}\left(\omega\left(\eta_{i}^{n} \eta_{k}^{n}+\eta_{k}^{n} \eta_{i}^{n}\right)+\omega\left(\left(\eta_{k}^{n}\right)^{2}\right)\right) .
\end{gathered}
$$

By using the translation invariance of $\omega$, the same method as above gives:

$$
\omega\left(S_{n}^{\prime \prime 2}\right) \leqq \sum_{x=-\infty}^{+\infty}\left|\omega\left(A \tau_{x} A\right)\right|\left(0\left(\frac{k q}{2 n+1}\right)+0\left(\frac{k q}{2 n+1}\right)+0\left(\frac{q}{2 n+1}\right)+0\left(\frac{p+q}{2 n+1}\right)\right)
$$

yielding (4.8). Together with (4.6) and (4.7) this proves Observation 1.

Observation 2:

$$
\lim _{n \rightarrow \infty}\left|\omega\left(e^{i t S_{n}^{\prime}(A)}\right)-\left[\omega\left(e^{i \iota_{0}^{\prime \prime}(A)}\right)\right]^{k(n)}\right|=0
$$

The basic idea consists of approximating $A$ by local observables $A_{N}$. This enables us to use the properties of the cluster function $\alpha_{N}^{\omega}($.$) , which are in fact the essential$ ingredients in the proof of this observation.

By $(\mathbf{C L} 2)$ we may choose a sequence $\left(A_{N}\right)_{N \in \mathbb{N}_{0}}$ such that

$$
\lim _{n \rightarrow \infty} N^{1+\varepsilon_{1}}\left\|A-A_{N}\right\|<\infty \text { for an } \varepsilon_{1}>0 .
$$

Define the subsequence $\left(A_{N(n)}\right)_{n \in \mathbb{N}_{0}}$ by $N(n)=[q(n) / 3]$, then

$$
\begin{aligned}
\left|\omega\left(e^{i t S_{n}^{\prime}(A)}-e^{i t S_{n}^{\prime}\left(A_{N(n)}\right)}\right)\right| & \leqq\left\|e^{i t S_{n}^{\prime}(A)}-e^{i t S_{n}^{\prime}\left(A_{N(n)}\right)}\right\| \\
& =\left\|\int_{0}^{t} d s e^{i S S_{n}^{\prime}\left(A_{N(n)}\right)}\left(S_{n}^{\prime}(A)-S_{n}^{\prime}\left(A_{N(n)}\right)\right) e^{i(t-s) S_{n}^{\prime}(A)}\right\|, \\
& \leqq t\left\|S_{n}^{\prime}(A)-S_{n}^{\prime}\left(A_{N(n)}\right)\right\| \\
& \leqq t k\left\|\xi_{0}^{n}(A)-\xi_{0}^{n}\left(A_{N(n)}\right)\right\| \\
& \leqq \frac{t k p}{(2 n+1)^{1 / 2}}\left\|A-A_{N(n)}\right\| \\
& =t 0\left((2 n+1)^{1 / 2}\left\|A-A_{[(q(n) / 3)]}\right\|\right) \\
& =t 0\left((\log 2 n+1) q(n) \cdot\left\|A-A_{[(q(n) / 3)]}\right\|\right) \\
& \leqq t 0\left(\left(\frac{q(n)}{3}\right)^{1+\varepsilon_{1} / 2}\left\|A-A_{[(q(n) / 3)]}\right\|\right) .
\end{aligned}
$$


Hence by (CL2) we obtain

$$
\lim _{n \rightarrow \infty}\left|\omega\left(e^{i t S_{n}^{\prime}(A)}\right)-\omega\left(e^{i t S_{n}^{\prime}\left(A_{N(n)}\right)}\right)\right|=0 .
$$

Analogously

$$
\begin{aligned}
& \left|\left[\omega\left(e^{l t \xi_{0}^{n}(A)}\right)\right]^{k(n)}-\left[\omega\left(e^{i t \xi_{0}^{n}\left(A_{N(n)}\right)}\right)\right]^{k(n)}\right| \\
& \quad=\left|\sum_{j=1}^{k}\left[\omega\left(e^{l t \xi_{0}^{n}(A)}\right)\right]^{k-j} \cdot\left[\omega\left(e^{i t \xi_{0}^{n}\left(A_{N(n)}\right)}\right)\right]^{j-1}\right| \cdot\left|\omega\left(e^{i t \xi_{0}^{n}(A)}\right)-\omega\left(e^{i t \xi_{0}^{n}\left(A_{N(n)}\right)}\right)\right| \\
& \quad \leqq k \cdot\left\|e^{t t \xi_{0}^{n}(A)}-e^{l t \xi_{0}^{n}\left(A_{N(n)}\right)}\right\| \leqq t k\left\|\xi_{0}^{n}(A)-\xi_{0}^{n}\left(A_{N(n)}\right)\right\| .
\end{aligned}
$$

Therefore

$$
\lim _{n \rightarrow \infty}\left|\left[\omega\left(e^{l t \xi_{0}^{n}(A)}\right)\right]^{k(n)}-\left[\omega\left(e^{l t \xi_{0}^{n}\left(A_{N(n)}\right)}\right)\right]^{k(n)}\right|=0 .
$$

Finally if we show that

$$
\lim _{n \rightarrow \infty}\left|\omega\left(e^{i t S_{n}^{\prime}\left(A_{N(n)}\right)}\right)-\left[\omega\left(e^{i t \xi_{0}^{n}\left(A_{N(n)}\right)}\right)\right]^{k(n)}\right|=0,
$$

then together with (4.9) and (4.10) Observation 2 is proved. But as

$\left[\xi_{i}^{n}\left(A_{N(n)}\right), \xi_{j}^{n}\left(A_{N(n)}\right)\right]=0$ for all $i, j$ one easily calculates

$$
\begin{aligned}
& \left|\omega\left(e^{i t S_{n}^{\prime}\left(A_{N(n)}\right)}\right)-\left[\omega\left(e^{i t \xi_{0}^{n}\left(A_{N(n)}\right)}\right)\right]^{k(n)}\right| \\
& =\left|\omega\left(\prod_{j=0}^{k-1} e^{i t \epsilon_{j}^{n}\left(A_{N(n)}\right)}\right)-\left[\omega\left(e^{i t \xi_{0}^{n}\left(A_{N(n)}\right)}\right)\right]^{k(n)}\right|
\end{aligned}
$$

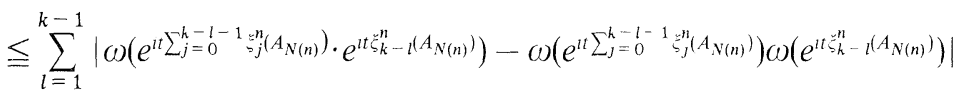

$$
\begin{aligned}
& \leqq \sum_{l=1}^{k-1} \alpha_{(k-l)(p+q)+2 N(n)}\left(\frac{q(n)}{3}\right) \\
& \leqq k \cdot \alpha_{k(p+q)}\left(\frac{q(n)}{3}\right) \quad \text { (Property 4.3) } \\
& \leqq(2 n+1)^{1 / 2} \alpha_{(2 n+1)}\left(\frac{(2 n+1)^{1 / 2}}{3 \log (2 n+1)}\right) \\
& \leqq(2 n+1)^{1 / 2} \alpha_{(2 n+1)}\left((2 n+1)^{1 / 2-\delta}\right) \quad \text { (Property 4.4), }
\end{aligned}
$$

and (4.11) follows by (CL5).

Observation 3:

$$
\lim _{n \rightarrow \infty}\left[\omega\left(e^{i t \xi_{0}^{n}(A)}\right)\right]^{k(n)}=e^{-(1 / 2) t^{2} S_{\omega)}(A, A)} .
$$

Define

$$
f_{n}(t)=\omega\left(e^{n t t_{0}^{n}}\right) .
$$

By Taylors asymptotic expansion, there exists a $c_{n}(t) \in[0, t]$ such that 


$$
\begin{aligned}
f_{n}(t) & =f_{n}(0)+f_{n}^{\prime}(0) t+\frac{f_{n}^{\prime \prime}(0)}{2} t^{2}+\frac{f_{n}^{\prime \prime \prime}(0)}{3 !} t^{3}+\frac{f_{n}^{(i v)}\left(c_{n}(t)\right)}{4 !} t^{4} \\
& =1-\omega\left(\left(\xi_{0}^{n}\right)^{2}\right) \frac{t^{2}}{2}+\omega\left(\left(\xi_{0}^{n}\right)^{3}\right) \frac{(i t)^{3}}{3 !}+\omega\left(e^{i c_{n}(t) \xi_{0}^{n}} \xi_{0}^{4}\right) \frac{(i t)^{4}}{4 !} .
\end{aligned}
$$

Furthermore, by (CL3):

$$
\begin{aligned}
\omega\left(\left(\xi_{0}^{n}\right)^{2}\right) & =\frac{p}{2 n+1} \cdot \frac{1}{p_{x, y=1}} \sum^{p} \omega\left(\tau_{x}(A) \tau_{y}(A)\right) \\
& =0\left(\frac{1}{k}\right) s_{p}(A, A)=0\left(\frac{1}{k}\right),
\end{aligned}
$$

and by (CL4):

$$
\begin{aligned}
\omega\left(\left(\xi_{0}^{n}\right)^{4}\right) & =\frac{p^{2}}{(2 n+1)^{2}} \cdot \frac{1}{p^{2}} \sum_{x, y, z . \omega=1}^{p} \omega\left(\tau_{x}(A) \tau_{y}(A) \tau_{z}(A) \tau_{\omega}(A)\right) \\
& =0\left(\frac{1}{k^{2}} \cdot p^{1-\varepsilon_{2}}\right)=0\left(\frac{1}{k^{1+\varepsilon_{2}^{\prime}}}\right) .
\end{aligned}
$$

Also, by Schwarz inequality:

$$
\begin{aligned}
\omega\left(\left(\xi_{0}^{n}\right)^{3}\right) & \leqq\left(\omega\left(\xi_{0}^{2}\right) \cdot \omega\left(\xi_{0}^{4}\right)\right)^{1 / 2} \\
& \leqq 0\left(\frac{1}{k^{1+\varepsilon_{3}}}\right)
\end{aligned}
$$

and

$$
\begin{aligned}
\left|\omega\left(e^{i c \xi_{0}^{n}}\left(\xi_{0}^{n}\right)^{4}\right)\right| & =\left|\omega\left(\left(\xi_{0}^{n}\right)^{2} e^{t c \xi_{0}^{n}}\left(\xi_{0}^{n}\right)^{2}\right)\right| \\
\leqq & \left|\omega\left(\left(\xi_{0}^{n}\right)^{4}\right)\right|=0\left(\frac{1}{k^{1+\varepsilon_{2}^{\prime}}}\right) \text { for all } c \in \mathbb{R} .
\end{aligned}
$$

Hence we have shown that

$$
\omega\left(e^{l t \xi_{0}^{n}(A)}\right)=1-\frac{1}{2} t^{2} s_{p(n)}(A, A) 0\left(\frac{1}{k(n)}\right)+0\left(\frac{1}{k(n)^{1+\varepsilon}}\right) .
$$

Therefore

$$
\lim _{n \rightarrow \infty}\left[\omega\left(e^{i t \xi_{0}^{n}(A)}\right)\right]^{k(n)}=e^{-(1 / 2) t^{2}} \lim _{n \rightarrow \infty} s_{p(n)}(A, A)=e^{-(1 / 2) t^{2} s_{\omega}(A . A)},
$$

proving Observation 3 .

For $v \geqq 2$ one proceeds along the same lines. In $\Lambda_{n}=[-n, n]^{v}$ we consider the small cube $\Gamma_{p}=[-n,-n+p-1]^{v}$ Define

and

$$
\xi_{0}^{n}(A)=\frac{1}{\left|\Lambda_{n}\right|^{1 / 2}} \sum_{x \in \Gamma_{p}} \tau_{x}(A)
$$

$$
\xi_{i}^{n}(A)=\tau_{i(p+q)}\left(\xi_{0}^{n}(A)\right)
$$

with $i=\left(i_{1}, \ldots, i_{v}\right) \in Z^{v}$ such that $0 \leqq i_{j} \leqq k-1$. 
Analogously as for $y=1$ one proves:

$$
\begin{aligned}
& \text { Observation 1: } \lim _{n \rightarrow \infty}\left|\omega\left(e^{i t A^{n}}\right)-\omega\left(e^{i t \sum_{i} \xi_{1}^{n}(A)}\right)\right|=0, \\
& \text { Observation 2: } \lim _{n \rightarrow \infty}\left|\omega\left(e^{t \sum_{i} \xi_{l}^{n}(A)}\right)-\left[\omega\left(e^{t \xi_{0}^{n}(A)}\right)\right]^{k^{n}}\right|=0, \\
& \text { Observation 3: } \lim _{n \rightarrow \infty}\left[\omega\left(e^{n \xi_{0}^{n}(A)}\right)\right]^{k^{n}}=e^{-(1,2) t^{2} s_{\omega}(A, A)}
\end{aligned}
$$

Obviously the theorem follows from these observations. Finally we prove the bound $(*)$

$$
\left\|\left[e^{i A}, B\right]\right\| \leqq\|[A, B]\|
$$

for all $A=A^{*}$ and $B$ elements of a $C^{*}$-algebra. It is a direct consequence of the more general formula:

$$
\left[e^{i A}, B\right]=i \int_{0}^{1} d t e^{i t A}[A, B] e^{i(1-t) A},
$$

which is easily seen to hold as follows:

$$
e^{i s B} e^{i A} e^{-i s B}=e^{i s[B, .]}\left(e^{i A}\right)=e^{i(\exp i s[B . .](A))}=e^{i\left(A+i s[B . A]+0\left(s^{2}\right)\right)} .
$$

It follows that

$$
\begin{aligned}
& \left.\frac{d}{d s}\left(e^{i s B} e^{i A} e^{-i s B}\right)\right|_{s=0} \\
& \quad=\left(\int_{0}^{1} d t e^{i t\left(A+i s[B, A]+0(s)^{2}\right)}(-[B, A]+0(s)) e^{i(1-t)\left(A+i s[B, A]+O(s)^{2}\right)}\right)_{s=0} \\
& \quad=\int_{0}^{1} d t e^{i t A}[A, B] e^{i(1-t) A} .
\end{aligned}
$$

On the other hand

$$
\left.\frac{d}{d s}\left(e^{i s B} e^{i A} e^{-i s B}\right)\right|_{s=0}=i B e^{i A}-i e^{i A} B=-i\left[e^{i A}, B\right],
$$

and $(* *)$ follows immediately.

\section{Example}

Interesting examples which should be studied are the equilibrium states of quantum spin systems. One should expect that for sufficiently short-ranged interactions the spin system has normal fluctuations. However, a rigorous approach to this problem requires a detailed analysis of the cluster properties of the equilibrium states, which lies beyond the purpose of this paper.

In this section we give a class of systems $\left(\mathscr{B}, \mathscr{B}_{0}, \omega\right)$ for which the conditions (CL1-CL5) are readily verified. These systems can be seen as a quantum analogue example of the finite dependent sequences in the classical theory, i.e. the clusterfunction $\alpha^{\omega}(d)$ has compact support. 
We fix the triplet $\left(\mathscr{B}, \mathscr{B}_{0}, \omega\right)$ as follows: $\mathscr{B}$ and $\mathscr{B}_{\Lambda}$ are respectively defined as the CCR-C*-algebras (Sect. 2) $\overline{W(\overline{H, \sigma})}$ and $\overline{W\left(H_{\Lambda}, \sigma\right)}$ with

$$
\begin{aligned}
& H=L^{2}(Z)=\left\{\left.\phi\left|\phi: Z \rightarrow \mathbb{C} ; \sum_{n=-\infty}^{+\infty}\right| \phi(n)\right|^{2}<\infty\right\}, \\
& H_{\Lambda}=L^{2}(\Lambda) \text { with } \Lambda \text { a finite subset of } \mathbb{Z}, \\
& \sigma(\phi, \psi)=\operatorname{Im}(\phi, \psi) \text { with } \\
& (\phi, \psi)=\sum_{n=-\infty}^{+\infty} \bar{\phi}(n) \psi(n) ; \phi, \psi \in L^{2}(\mathbb{Z}) .
\end{aligned}
$$

$\mathscr{B}_{0}$ is the subalgebra of local observables defined by $\mathscr{B}_{0}=\cup_{\Lambda} \mathscr{B}_{\Lambda}$. The representation $\left(\tau_{x}\right)_{x \in Z}$ of the translation group $\mathbb{Z}$ is defined by

$$
\tau_{x} W(\phi)=W\left(U_{x} \phi\right) \quad \text { with }\left(U_{x} \phi\right)(y)=\phi(y-x) ; \quad \psi \in L^{2}(Z) .
$$

Although $\mathscr{B}$ is not a quasi-local algebra in the strict sense [20], the theory developed in Sects. 3 and 4 can be formulated analogously. The Fourier-transform $\mathscr{F}: L^{2}(Z) \rightarrow$ $L^{2}([0,2 \pi])$ is defined by

$$
(\mathscr{F} \phi)(k)=\hat{\phi}(k)=\sum_{n=-\infty}^{+\infty} e^{-i k n} \phi(n) .
$$

For any function $f \in L^{2}(Z)$ such that $\hat{f} \geqq 0$ we can define the positive operator $A_{f}$ and the associated translation invariant quasi-free state $\omega_{f}$ by:

with

$$
A_{f}: L^{2}(Z) \rightarrow L^{2}(Z): \phi \rightarrow A_{f} \phi=f * \phi
$$

and

$$
(f * \phi)(j)=\sum_{n=-\infty}^{+\infty} f(j-n) \phi(n)
$$

$$
\omega_{f}(W(\phi))=e^{-1 / 4|\phi|^{2}} \cdot e^{-1 / 2\left(\phi, A_{f} \phi\right)} .
$$

Now we show that if the function $f$ has compact support, e.g. $\operatorname{supp} f=\Lambda_{f}$, then the system $\left(\mathscr{B}, \mathscr{B}_{0}, \omega_{f}\right)$ defined above satisfies the conditions $(\mathrm{CL} 1 \rightarrow 5)$.

Obviously the conditions $(\mathrm{CL} 1,2)$ on the subalgebra $\mathscr{B}_{0}$ are satisfied. To verify the conditions $(\mathrm{CL} 3,4,5)$ on the state $\omega_{f}$, observe that $\left(\phi, A_{f} \psi\right)=0$ for $\phi \in L^{2}(\Lambda)$, $\psi \in L^{2}(\hat{\Lambda})$ with $\alpha(\Lambda, \hat{\Lambda})>\left|\Lambda_{f}\right| / 2$. Hence

$$
\omega_{f}(W(\phi) W(\psi))=\omega_{f}(W(\phi+\psi))=\omega_{f}(W(\phi)) \omega_{f}(W(\psi))
$$

implying for example that $\alpha^{\omega f}(d)=0$ for $d>\left(\left|\Lambda_{f}\right| / 2\right)$. Obviously this example can be generalized to higher dimensions.

Acknowledgements. The authors would like to thank Professor A. Verbeure for introducing us to the problem and for many suggestions. We thank also M. Fannes and B. Nachtergaele for many stimulating discussions.

\section{References}

1. Lanford, O. E., Ruelle, D.: J. Math. Phys. 13, 194 (1969)

2. Hepp, K., Lieb, E. H.: Ann. Phys. 76, 360 (1973) 
3. Hepp, K., Lieb, E. H.: Helv. Phys. Acta 46, 573 (1974)

4. Wreszinski, W. F.: Helv. Phys. Acta 46, 844 (1974)

5. Goderis, D., Verbeure, A., Vets, P.: Non-commutatıve Central Limits. J. Prob. Theory Rel. Fields (to appear)

6. Goderis, D., Verbeure, A., Vets, P.: Theory of fluctuations and small oscillations for quantum lattice systems. J. Math. Phys. 29, 2581 (1988)

7. Giri, N., von Waldenfels, W.: Z. Wahrscheınlichkeitstheorie Verw. Geb. 42, 135 (1978)

8. Accardi, L., Bach, A.: Z. Warscheinlichkeitstheorie Verw. Geb. 68, 393 (1985)

9. Cushen, C. D., Hudson, R. L.: J. Appl. Probability 8, 454 (1971)

10. Bernstein, S.N.: Math. Ann. 97, 1 (1926)

11. Ibramigov, I. A., Linnick, Yu.V.: Independent and stationary sequences of Random Variables, Wolters--Noordhoff, 1971

12. Haag, R., Kadison, R. V., Kastler, D.: Commun. Math. Phys. 33, 1 (1973)

13. Manuceau, J., Sirugue, M., Testard, D., Verbeure, A.: Commun. Math. Phys. 32, 231 (1973)

14. Manuceau, J., Sirugue, M., Rocca, F., Verbeure, A.: Quasi-free States; Cargèse Lecture Notes in Physics, Vol. 4, Kastler D. (ed.), New-York: Gordon Breach 1970

15. Manuceau, J.: Ann. Inst. H. Poincaré 2, 139 (1968)

16. Bratteli, O., Robinson, D. W.: Operator algebras and quantum statistical mechanics, Vol. 1 Berlin Heidelberg New York: Springer 1979

17. Lance, C.: Tensor products of $C^{*}$-algebras; $C^{*}$-algebras and their applications to statistical mechanics and quantum field theory. (Proc. Int. School of Phys. "Enrico Fermi". Amsterdam: North-Holland 1976

18. Fannes, M., Spohn, H., Verbeure, A.: J. Math. Phys. 21, 355 (1980)

19. Chung, K. L.: A Course in probability theory, New York: Academic Press 1968

20. Bratteli, O., Robinson, D. W.: Operator algebras and quantum statistical mechanics, Vol. II. Berlin Heidelberg, New York: Springer 1981

Communicated by H. Araki

Received July 13, 1988 
